The Israeli Journal of Aquaculture ISSN 0792-156X IJA.73.2021.1546544, 12 pages

CCBY-NC-ND-4.0 • https://doi .org/10.46989/001c.30754

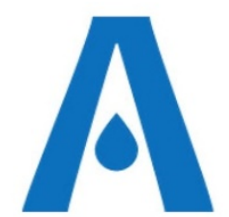

The $I J A$ is a peer-reviewed open-access, electronic journal, freely available without charge to users

Produced by the AquacultureHub non-profit Foundation Sale of IJA papers is strictly forbidden

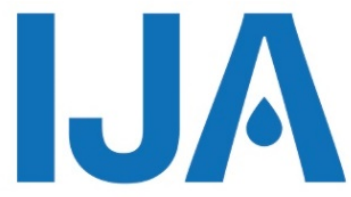

\title{
Variation of bacterial communities in black tiger shrimp (Penaeus monodon) with different growth performances
}

\author{
Yundong $\mathrm{Li}^{1,2,3,4}$, Falin Zhou ${ }^{1,2}$, Wenwen Zhang ${ }^{1}$, Qibin Yang ${ }^{1,2}$, Song Jiang ${ }^{1,2}$, \\ Jianhua Huang ${ }^{1,2}$, Lishi Yang ${ }^{1,2}$, Shigui Jiang ${ }^{1,2,4 *}$
}

${ }^{1}$ Key Laboratory of South China Sea Fishery Resources Exploitation and Utilization, Ministry of Agriculture and Rural Affairs, South China Sea Fisheries Research Institute, Chinese Academy of Fishery Sciences, Guangzhou 510300, China

2 Tropical Aquaculture Research and Development Center, South China Sea Fisheries Research Institute, Chinese Academy of Fishery Sciences, Sanya 572018, China

3 Key Laboratory of Tropical Hydrobiology and Biotechnology of Hainan Province, Hainan Aquaculture Breeding Engineering Research Center, College of Marine Sciences, Hainan University, Haikou, Hainan 570228, China

${ }^{4}$ Hainan Yazhou Bay Seed Laboratory, Sanya, 572025, China

Key words: Penaeus monodon, shrimp, intestinal bacteria, growth, 16S rRNA sequencing

\begin{abstract}
The intestinal bacteria of aquatic animals are closely related to their health and growth status. Penaeus monodon has become an important aquaculture species in the world because of its high nutritional value and market to demand. However, knowledge of bacterial communities on the growth phenotype of $P$. monodon is still limited. In this research, the bacterial community were comprehensively analyzed by using Miseq sequencing technology and bioinformatics. The results indicated that 29 phyla and 468 genera were identified by the taxonomic sequence. The data showed that the composition of microbial community was statistically different to fast-growing shrimps and slow-growing shrimps. There were significant differences in intestinal bacteria at the genus level. Lactococcus, Limnothrix, and Arthrospira had more abundance in the gut of fast-growing shrimps, which may promote the growth of $P$. monodon by affecting enzyme activities and improving immune response. Nautella, Shimia, Pseudoalteromonas, Aliiroseovarius, Albimonas, Tessaracoccus had more abundance in the gut of slow-growing shrimps. Nautella had the ability to express virulence by stimulated by environmental factors, thereby increasing the susceptibility to infection, which may have a negative impact on healthy growth of shrimps. Pseudoalteromonas, Aliiroseovarius and Shimia were related to the pathogenicity of shrimp, which may be an important factor affecting the growth of shrimp. Overall, this study demonstrates that the bacterial community associated with $P$. monodon was variation on different growth performance. These results can provide information on microbiome characteristics involved in the asynchronous growth of shrimp.
\end{abstract}

*Corresponding author: Shigui Jiang, Tel.: +86-20-89108338, Fax: +86-20-84451442, e-mail: jiangsg@21cn.com, South China Sea Fisheries Research Institute, Chinese Academy of Fishery Sciences, 231 Xingang West Road, Guangzhou 510300, China. 


\section{Introduction}

With the increase in shrimp consumption demand, the decline in wild production forced artificial culture to become the main source of shrimp production (Lebel et al. 2010). Due to its high market price and market to demand, the intensive culture of $P$. monodon is expanding year by year. However, in recent years, the industry has faced some major challenges, such as the lack of stable water quality control and disease management (Rungrassamee et al. 2014); In addition, there is still lack of research on P. monodon especially on the aspect of microbiology including intestinal bacteria, and most previous research focused on the field of population biology, breeding, and genetic diversity (Nahavandi et al. 2013).

It has been recognized that intestinal bacteria is closely related to the growth and health of the host, intestinal bacteria can improve intestinal digestion and absorption function, promote intestinal nutrition balance, which is beneficial to the health of the host. Normal intestinal bacteria metabolites are conducive to killing pathogenic bacteria and inhibiting the growth of pathogens. In addition, intestinal bacteria also maintain the stability of intestinal barrier and stimulate the immune system (Xiong et al. 2017). Previous researches have indicated that shrimp bacterial community may also have some beneficial effects on shrimp (Chaiyapechara et al. 2012). Therefore, taxonomic study and functional analysis of bacterial communities are essential for the discovery of potential pathogenic, probiotics and other microbes that have a profound impact on health and overall host physiological function (Wu et al. 2010).

In recent years, various researches have been carried out on intestinal bacteria in aquaculture animals, such as Eriocheir Sinensis, grass carp, and Holothuria scabra (Wang et al. 2019). The intestinal bacteria of Litopenaeus vannamei and $P$. monodon have been well studied (Rungrassamee et al. 2013; Zeng et al. 2017), and most of the reports on $P$. monodon focus on the impact of nutritional diets and diseases on intestinal bacteria (Rungrassamee et al. 2014). However, the study of bacterial community related to the growth of $P$. monodon is still limited. Therefore, a systematic comparison of the characteristics of intestinal bacteria between FG shrimps and SG shrimps is essential for screening probiotics, which will facilitate our understanding of the relationship between intestinal bacteria and growth performance.

Hence, we used high-throughput sequence to study intestinal bacteria between fastgrowing and slow-growing individuals. Our main goal were to reveal the composition and function of the intestinal bacteria between fast-growing and slow-growing individuals of $P$. monondon, and then to explore the relationship between shrimp growth and intestinal bacteria. To our knowledge, this study was the first high-throughput sequencing study on the relationship between intestinal bacteria and growth of $P$. monodon with the same genetic background.

Sample collection

\section{Materials and Methods}

The study was approved by the Animal Care and Use Committee of SCSFRI, Chinese Academy of Fishery Sciences (SCSFRI96-253). Healthy shrimps was obtained from the SCSFRI (South China Sea Fisheries Research Institute) in Shenzhen (Guangdong, China). By artificial insemination, we obtained shrimp larvae from the same pair of parents, this batch of shrimp was cultured in the same pond. Therefore, in this study, this batch of samples had the same genetic background and were grown in the same environment. The shrimps were maintained at pool containing aerated filtered seawater (salinity, 30 31\%o; $\mathrm{pH}, 7.9 \sim 8.2$; temperature, $27 \sim 31^{\circ} \mathrm{C}$ ), and the shrimps were fed with commercial diets.

After 90 days cultured, we fished shrimps from ponds and weighed, selecting individuals who were larger in size and those who were smaller. Finally, 24 shrimp were used for sampling (12 large, 12 small) to obtain intestinal tissue separately, and each intestine sample is mixed with 3 healthy shrimps' intestine. The shrimps' surface was sterilized with $75 \%$ ethanol and the intestine was aseptically dissected. The samples were immediately frozen in liquid nitrogen, and stored at $-80^{\circ} \mathrm{C}$ until DNA extraction was 
required. FG stands for the fast-growing group and SG stands for the slow-growing group. The body length and body weight of FG respectively were $13.83 \pm 0.66 \mathrm{~cm}, 36.86 \pm 5.83$ $\mathrm{g}$, and the body length and body weight of SG respectively were $9.61 \pm 0.60 \mathrm{~cm}, 12.24 \pm$ $2.16 \mathrm{~g}$ (Figure. 1).
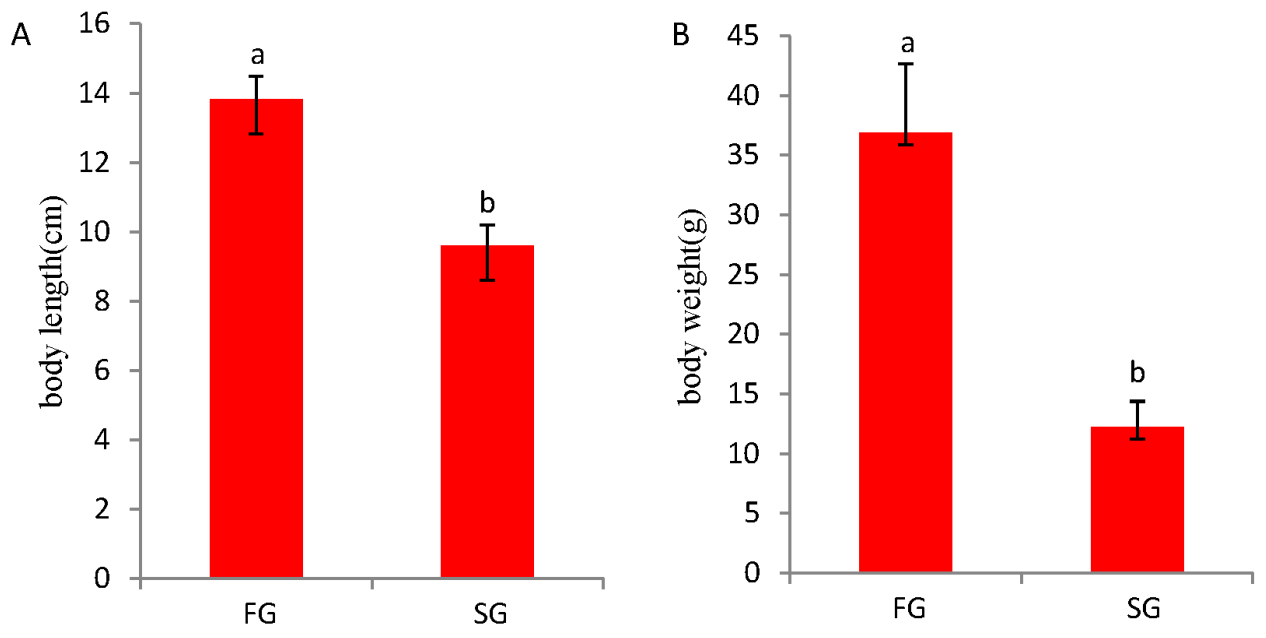

Figure 1 The comparison of growth phenotypes of Penaeus monodon comes from fast-growing group (FG) and slow-growing group (SG).

DNA extraction and Illumina sequencing.

In this study, a total of eight sequence libraries were constructed, including four libraries from the FG (F1, F2, F3, F4) and four other libraries from the SG (S1, S2, S3, S4). All DNAseq programs were performed in the Majorbio Company (Shanghai, China). Illumina truseqtm RNA sample preparation kit (Illumina, USA) was used to construct library, and checked in Agilent 2100 biological analyzer (Agilent, 2100). The total DNA content was determined by $1 \%$ agarose gel electrophoresis and used for subsequent microbial community analysis, the concentration and the purity were determined by Nanodrop ND 2000 spectrophotometer (SEM Fisher technology, Waltham, Massachusetts).

The V3-V4 hypervariable regions of 16S rRNA genes were amplified by PCR for 29 cycles, finally, primers 338F (ACTCCTACGGGAGGCAGCAG) and 806R (GGACTACHVGGGTWTCTAAT) with different barcodes were used to extend at $72{ }^{\circ} \mathrm{C}$ for 10 min. The PCR products were detected by agarose gel containing $2 \%$ ethidium bromide and purified by using AxyPrep DNA Gel Extraction Kit (Axygen Biosciences, California), quantified using QuantiFluor ${ }^{\mathrm{TM}}$-ST (Promega, USA). The purified amplicons were combined equimolar and sequenced on Illumina-miseq platform $(2 \times 300 \mathrm{bp})$ according to the standard procedure.

Sequence analysis processing

The original fastq sequence files were demultiplexed and filtered, and then merged by fastp and flash, which met the criterion listed below: (i) the reading segments were truncated at any site with an average quality score less than 20 on the 50 bp sliding window; (ii) the sequences of overlapping length more than $10 \mathrm{bp}$ were merged according to the overlapped sequences; (iii) the primers were accurately matched, two nucleotide mismatches were allowed, and the reading segments containing ambiguous bases were deleted.

Using uparse (version $7.0 \mathrm{http}: / /$ drive5.com/uparse/), the similarity in operational taxonomic units (OTUs) was up to $97 \%$, and the chimeric sequences were identified and removed by uchime. Based on the 70\% confidence threshold of Silva (ssu115) 16S rRNA database, the RDP classification algorithm (version 2.2 
http://sourceforge.net/projects/rdp-classifier/) was used to analyze the classification of each 16S rRNA gene sequence in Silva 16S rRNA database (Amato et al. 2013).

Statistical analysis

The reflectance curve of each sample was plotted to determine the community number and sequence data onto each sample (Amato et al., 2013). The monthly software were used to calculate community diversity parameters including Shannon, Simpson and Shannon Wiener curve, community richness parameters (including Chao and ACE) and good's coverage index. (Schloss et al. 2011). Beta diversity measurements were calculated according to the method described (Jiang et al., 2013), and the Venn chart was determined based on the OTU level.

The differences between the two groups were analyzed by using Wilcoxon rank-sum test or Mann-Whitney $U$ test. The results of $P<0.05$ between the two groups were considered to be statistically significant. The differences of abundance were analyzed by using Non-parametric factorial Kruskal-Wallis sum-rank test. The results of LDA $<2$ between groups were considered to be statistically significant. All the results were the average of four samples' result (mean \pm SD).

Miseq sequencing and microbiome analysis

\section{Results}

In order to breed intestinal bacteria between FG and SG, the V3-V4 region of $16 \mathrm{~S}$ rRNA gene was sequenced by using Illumina Miseq platform. A total of 410,081 high-quality sequence readings were obtained, with an average of 51,260 readings ranging from 38,230 to 62,430 (Table 1). These sequences were aggregated into 816 OTUs with $97 \%$ sequence identity, and each library contained different OTUs ranging from 123 to 461 .Table 1 showed statistical estimates of readings, number of OTUs, coverage and species diversity and richness indices for each sample at a genetic distance of $3 \%$.

Diversity and richness indices were calculated for all FG and SG samples to show the complexity of each sample (Table 1). The Shannon index and Simpson index were in direct proportion to the community diversity index, ranging from 0.372135 to 2.640287 and 0.169225 to 0.899601 , respectively. The Chao index and ACE index represent the community richness, ranging from 188.15 to $466.24,184.35$ to 469.28 , respectively. The estimated good's coverage of each sample of sequence integrity was $>99.86 \%$ (from 0.998683 to 0.999915 ), which indicated that the coverage rate of bacteria in the samples was high and the sequence depth was reasonable, which met the needs of bacterial diversity analysis in samples.

Table 1 Richness and diversity indexes relative to each sample. OTUs were defined at the $97 \%$ similarity level.

\begin{tabular}{llllllll}
\hline Sample & Read & Coverage & Number & \multicolumn{4}{c}{ Alpha diversity } \\
\cline { 5 - 8 } ID & numbers & & of OUTs & Shannon & Simpson & ACE & Chao \\
\hline F1 & 40956 & 0.998732 & 127 & 0.624313 & 0.796093 & 224.3374 & 194.56 \\
F2 & 46477 & 0.999107 & 153 & 1.254975 & 0.553914 & 184.3575 & 188.15 \\
F3 & 60009 & 0.999915 & 246 & 1.784047 & 0.465129 & 247.3076 & 246.90 \\
F4 & 57072 & 0.998769 & 321 & 2.333637 & 0.237917 & 373.7325 & 374.625 \\
S1 & 53351 & 0.999186 & 160 & 1.802334 & 0.354169 & 195.2689 & 190.27 \\
S2 & 38230 & 0.998745 & 123 & 0.372135 & 0.899601 & 207.8508 & 192.16 \\
S3 & 51556 & 0.998683 & 319 & 2.423746 & 0.257548 & 360.9835 & 362.17 \\
S4 & 62430 & 0.999528 & 461 & 2.640287 & 0.169225 & 469.2892 & 466.24 \\
\hline
\end{tabular}

Compositions and structure of intestinal bacteria

Intestinal bacteria from all samples were studied and a Veen diagram was constructed to identify the dominant OTUs in both groups (Figure 2A). 312 OTUs $(n=4)$ are shared between $\mathrm{F}$ and $\mathrm{s}$, representing $38.23 \%$ of the total reads. FG owns 183 OTUs, representing $22.43 \%$ of the total number of reading. SG owns 321 OTUs, representing $39.34 \%$ of the total number of reading. According to the 16S rRNA gene sequence, 29 prokaryotes phyla 
were identified. Sequences that cannot be categorized are also specified as others. The relatively abundant phyla in the two groups were Proteobacteria $(69.19 \%)$, Firmicutes $(15.00 \%)$, Patescibacteria $(6.47 \%)$, Cyanobacteria $(3.08 \%)$, Bacteroidetes $(2.63 \%)$, Actinobacteria $(1.88 \%)$, Tenericutes $(1.19 \%)$ and others $(0.56 \%)$ (Figure 2 ).

(A)
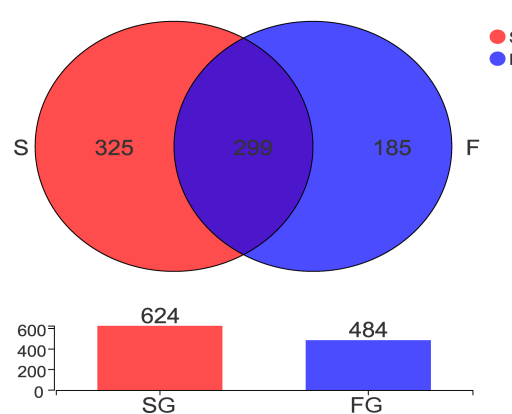

(C)

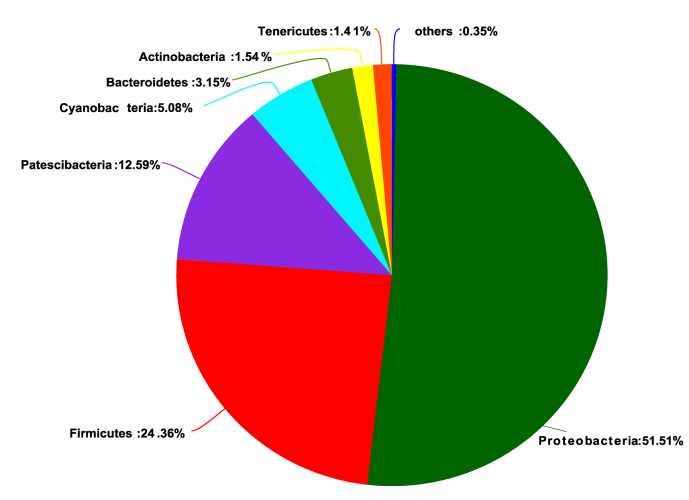

(B)

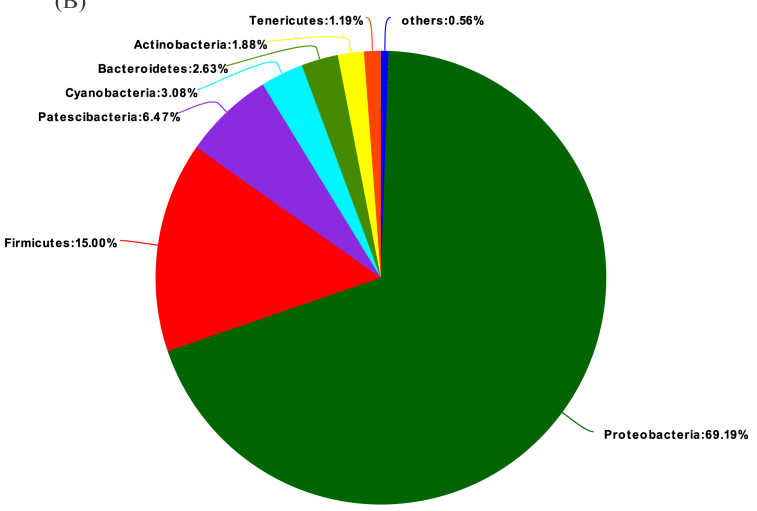

(D)

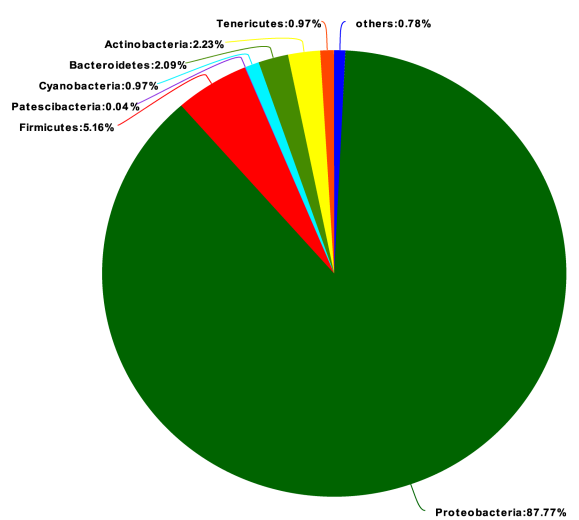

Figure 2 Comparison of OTUs in the two groups by Venn diagram (A) and the microbiota composition at phylum level (B, C, D). The bacterial community in all samples at phylum level (B). Microbiota composition of bacterial taxa at phylum level respectively in SG (C) and FG (D).

The dominant phyla in the two groups were Proteobacteria, Firmicutes, Patescibacteria, Cyanobacteria. There was no significant difference in the composition of dominant species between the FG and the SG. The dominant genera were Photobacterium, Staphylococcus, Nautella, Vibrio, Ruegeria. The dominant genera of the FG and the SG were significantly different (Figure 3). The dominant phyla were Proteobacteria $(F=57.05 \pm 36.27 \%, S=$ $88.19 \pm 9.18 \%, P=0.31)$, Firmicutes $(F=20.46 \pm 33.2 \%, S=5.16 \pm 9.23 \%, P=0.31)$, Patescibacteria $(\mathrm{F}=11.27 \pm 22.51 \%, \mathrm{~S}=0.04 \pm 0.03 \%, \mathrm{P}=0.88)$, Cyanobacteria $(\mathrm{F}=$ $4.89 \pm 5.59 \%, \mathrm{~S}=0.89 \pm 0.84 \%, \mathrm{P}=0.31$ ) (Figure 2). The dominant genera were Photobacterium $(F=41.93 \pm 48.26 \%, S=52.33 \pm 35.47 \%, P=0.66)$, Staphylococcus $(F=16.88 \pm 33.76 \%, S=0.01 \pm 0.01 \%, P=0.77)$, Nautella $(F=0.04 \pm 0.05 \%, S=12.06$ $\pm 14.84 \%, P=0.03)$, Vibrio $(F=1.42 \pm 1.86 \%, S=5.81 \pm 9.17 \%, P=0.66)$, Ruegeria $(\mathrm{F}=0.19 \pm 0.21 \%, \mathrm{~S}=5.15 \pm 8.68 \%, \mathrm{P}=0.06)$. 


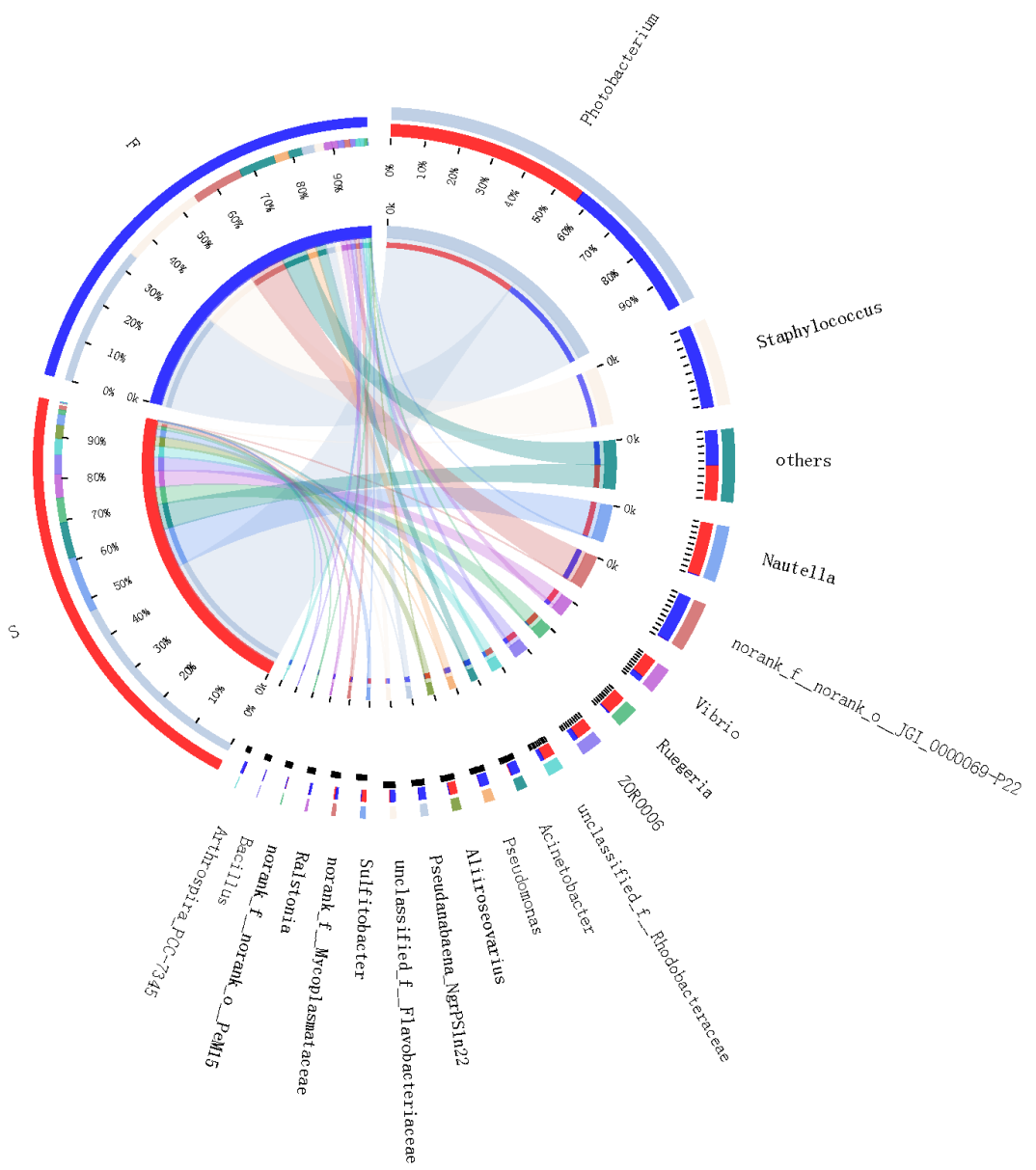

Figure 3 The microbiota composition at genus level.

Using Wilcoxon rank-sum test or Mann-Whitney $U$ test, Illumina miseq sequencing data showed that there was no significant difference in the relative abundance of intestinal bacteria between the FG and SG at the phylum level, but there was a significant difference between the FG and SG at the genus level (Figure 4). In the observed 468 identified genera, there were six genera (Nautella, Shimia, Pseudoalteromonas, Aliiroseovarius,Tessaracoccus, Albimonas) were identified as significantly different in the FG and SG. 

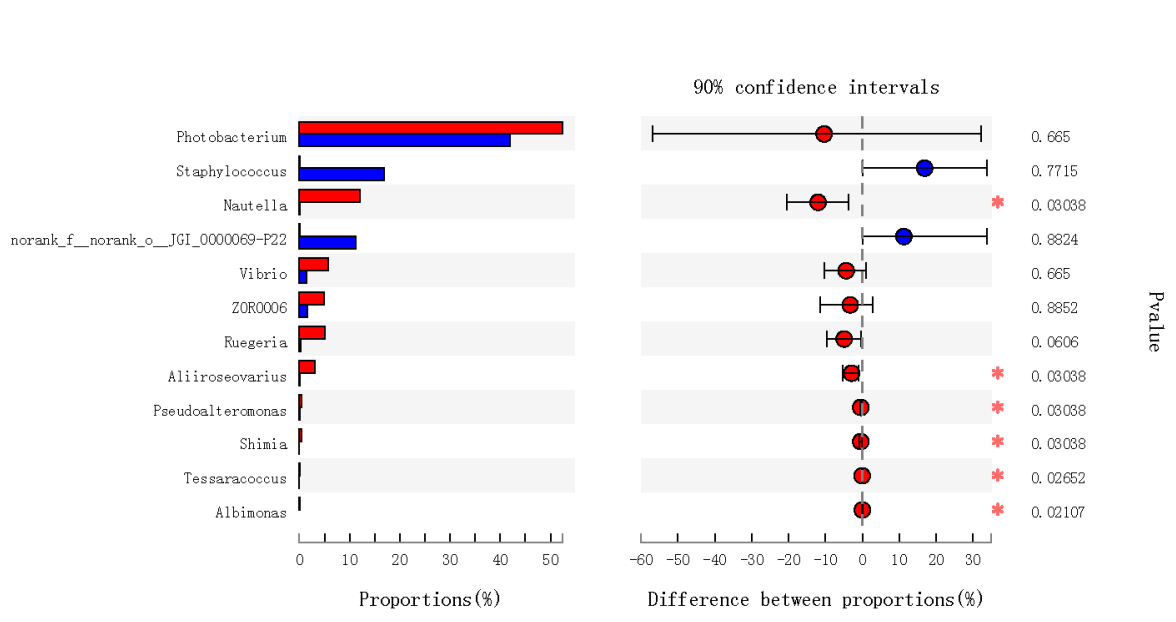

Figure 4 Comparison of bacterial abundances in fast-growing shrimp and slow-growing shrimp at the genus level. Wilcoxon rank-sum test bar plot on Genus level for intestinal bacterial. * stands for $\mathrm{P} \leq 0.05$.

Using non-parametric factorial Kruskal-Wallis sum-rank test, sequencing data demonstrated that Limnotrichales, Nostocales had more abundance in the intestine of FG shrimp at the order level, Rhodobacterales, Propionibacteriales, Kineosporiales had more abundance in the gut of SG shrimp (Figure 5). At the family level, Limnotrichaceae, Cyanobacteriaceae, Phormidiaceae, Simkaniaceae had higher abundance in the gut of FG shrimp, Pseudoalteromonadaceae, Rhodobacteraceae, Methyloligellaceae, Kineosporiaceae, Rubritaleaceae had higher abundance in the shrimp gut of SG than FG. At the genus level, Lactococcus, Limnothrix, Arthrospira, unclassified_f_Oligoflexaceae, Cyanobacterium_CLg1 had more abundance in the gut of FG, Pseudoalteromonas, Alteromonas, Nautella, unclassified_f_Rhodobacteraceae, Shimia, Ruegeria, Aliiroseovarius, Sulfitobacter, Albimonas, norank_f_Methyloligellaceae, Sphingobium, Halobacteriovorax, Ulvibacter, Tessaracoccus, Quadrisphaera had more abundance in the gut of SG (Figure 5). 


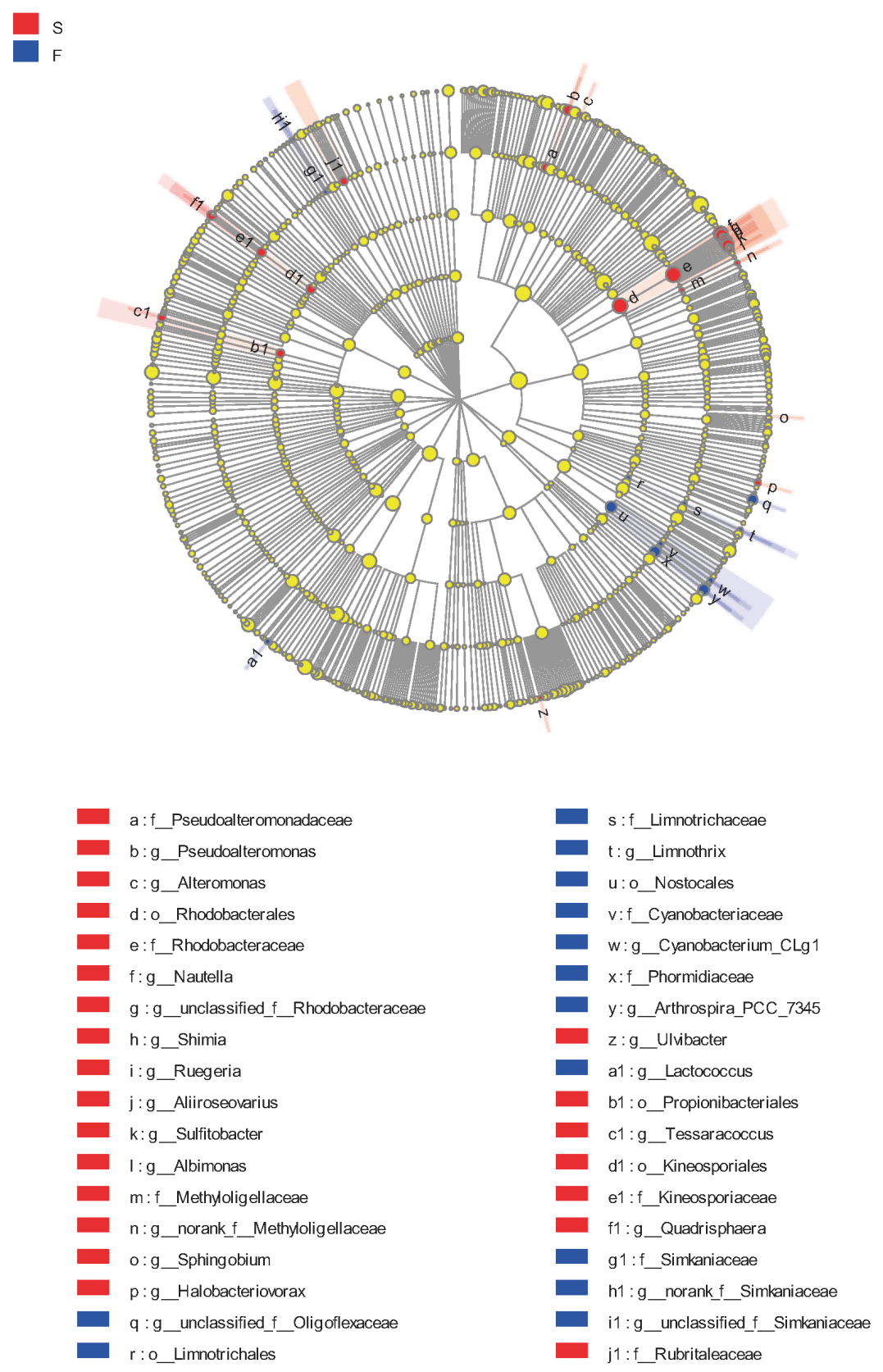

Figure 5 Comparison of intestinal bacterial abundance between fast-growing shrimp (F) and slow-growing shrimp (S) from the phylum level to the genus level .Different color nodes indicate microbial groups that are significantly enriched in the corresponding group and have a significant impact on the differences between groups; light yellow nodes indicate microbes that are not significantly different in different groups or have no significant impact on the differences between groups Taxa. 
Functional prediction of the intestinal bacteria

The putative functions of intestinal bacteria in FG and SG were illustrated by PICRUSt. By comparing the predicted genes with the eggnog databases, the genes were classified (Figure 6). The functional profiles of all groups are more similar than taxonomic profiles. The results of these COG functional classifications showed that the intestinal bacteria of shrimp maintained similar biological functions of those in the gut of both the FG and the SG.

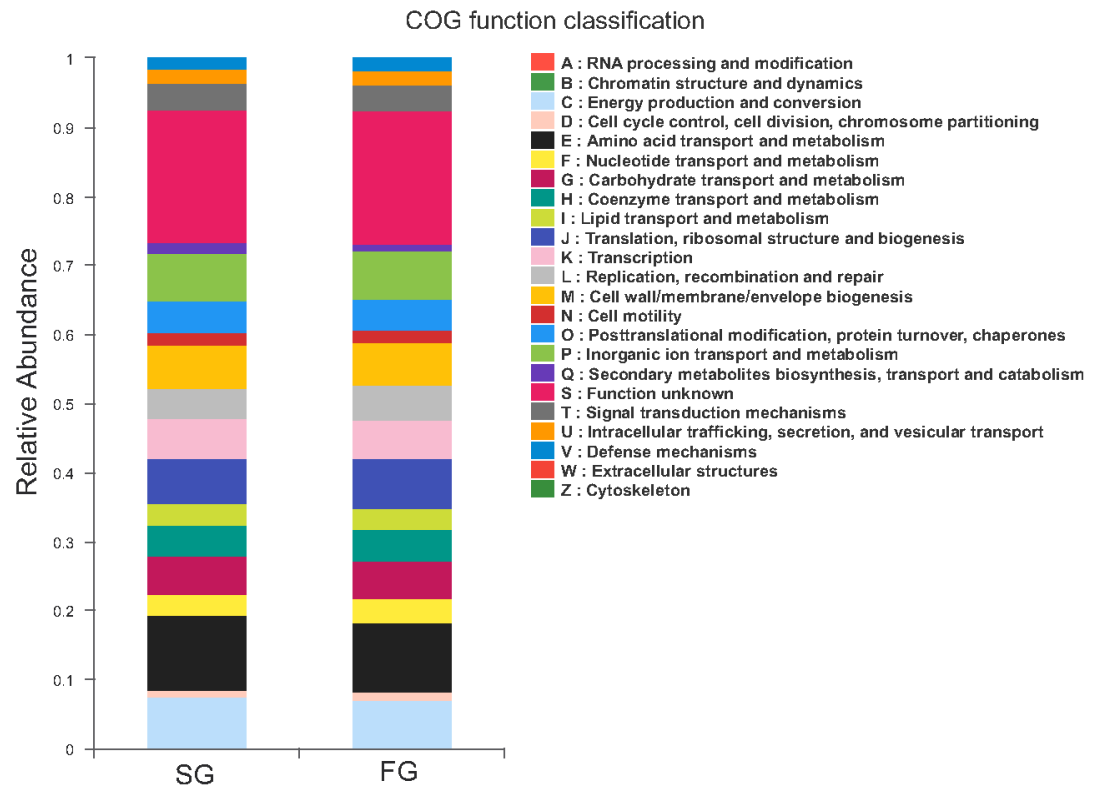

Figure 6 The COG function classification of the fast-growing group (FG) and slow-growing group (SG).

\section{Discussion}

Intestinal bacteria can regulate the intestinal environment and promote the balance of intestinal bacteria, and play a key role in the healthy growth of host (Li et al. 2013). Research had indicated that intestinal bacteria were vital host digestion and absorption, resistance to pathogens and immune regulation. However, the function of intestinal bacteria in $P$. monodon is still limited. High-throughput sequencing technology can accurately reflect the intestinal bacteria related to shrimp growth (Gao et al., 2019). In this research, we revealed the structure and function of intestinal bacteria of $P$. monodon by Miseq sequencing technology and bioinformatics. Simultaneously, we revealed that intestinal bacteria of FG shrimps differed SG shrimps.

As biomaterials, the gut contents of fish and shrimp had been widely used for bacterial analysis (Zeng et al. 2017). Note that gut contents reflect the combination of bacteria and resident in the gut, which may be different from gut samples or feces. However, whether there is such a difference in shrimp is worth further study.

Previous researches had indicated that Proteobacteria, Firmicutes, Bacteroidetes and Unclassified Bacteria, Planctomycetes and Actinobacteria were the main phyla in $P$. monodon, their relative abundance between shrimp gut at different developmental stages and living environment were different significantly (Rungrassamee et al. 2013; Angthong et al., 2020). In this research, Proteobacteria and Firmicutes were dominated phyla in $P$. monodon, which was consistent with previous researches (Chaiyapechara et al. 2012; Rungrassamee et al. 2014). It is worthy to note that the diversity of intestinal bacterial in SG is lower than that in FG at genus level, which can be explained by the relative abundance change of some bacteria. 
We found that Photobacterium was the most abundant genus in both groups. While we were interested in Nautella, which exhibited statistically significant differences between FG and SG. Nautella in gut was sensitive to environmental stresses such as salinity, ammonia, and nitrite stress (Zhang et al., 2016; Duan et al., 2018). When these factors change significantly, Nautella may cause diseases by expressing virulence, Zheng et al. (2017) shown that excessive accumulation of Nautella may change the health status of shrimp. Thus, the abundance of Nautella should be carefully monitored during shrimp culture, especially in the middle and late stages of shrimp farming, so as to avoid potential danger. In this study, we found that Nautella tends to be accumulated in the gut of SG, which was much higher than that in the FG. Therefore, the increase in Nautella in this study indicates that the intestinal bacteria of the SG may become disordered, and the shrimp may be in an unhealthy condition, leading to its slow growth.

Amoah et al. (2020) shown that Shimia were more abundant in the untreated group which grow slowly than in the probiotic-treated groups which grow faster. It is reported that Aliiroseovarius crassostreae was the pathogen of Roseovarius oyster disease (Duan et al. 2020) had shown that Pseudoalteromonas is an opportunistic pathogen, which might exacerbate spoilage by hydrolysing amino acids and proteins and decomposing lipids (Broekaert et al., 2013), it can cause high mortality in Portunus pelagicus (Duan et al., 2019). In present research, the abundances of Pseudoalteromonas, Aliiroseovarius and Shimia were significantly larger in the SG than another group, they may have adverse effects on the health of $P$. monodon, thereby retarding the growth of $P$. monodon.

Previous researches had shown that Lactococcus has been studied an important in activating the innate immune system to stimulate cytokine production, which regulates the immune response (Sun et al., 2018). It has been reported that depression of Lactococcus might result in the occurrence of white feces syndrome (Hou et al., 2018). We found that Lactococcus tends to be accumulated in the gut of FG, which can have a positive effect on digestive enzyme activities, growth performance and disease resistance in $L$. vannamei (Hou et al., 2018). Arthrospira had several positive effect on the physiological health of $L$. vannamei, such as immunomodulatory, antioxidant, anticancer, antimicrobial and antiviral activities (Macias-Sancho et al., 2014; Pilotto et al., 2019). In this study, Arthrospira tends to be accumulated in the gut of FG shrimps, it is likely that Arthrospira improve the immune response by reducing apoptosis and increasing the number of granular hemocytes, thereby affecting shrimp growth performance. So, we speculate that Lactococcus and Arthrospira can be used as a potential probiotic for the culture of $P$. monodon.

The intestinal bacterial communities were significantly different from FG and SG. Our study demonstrated that the growth performance of SG shrimps might be associated with decreased diversity of the intestinal bacterial community. Lactococcus, Limnothrix, Arthrospira, Cyanobacterium may promote the growth of $P$. monodon. Nautella, Shimia, Pseudoalteromonas, Aliiroseovarius, Tessaracoccus, Albimonas may retard the growth of $P$. monodon. These above results may be useful for the use of microbial community characteristics to guide the culture of $P$. monodon.

In conclusion, the microbiota relationship between the FG and SG in the same cultured environment was established. This study showed that although the profiles of microbial community in shrimp intestine was different, there was a closely relationship between the shrimp growth and their microbial communities. Moreover, this research indicated that the physiology of shrimp is closely related to the changes of bacteria in shrimp intestines. At the genus level, Lactococcus, Arthrospira, Limnothrix, Cyanobacterium had more abundance in the gut of FG shrimps. Lactococcus and Arthrospira may promote the growth of $P$. monodon respectively by the effect on the enzyme activities and immune response. Nautella, Shimia, Pseudoalteromonas, Aliiroseovarius, Albimonas, Tessaracoccus had more abundance in the gut of SG shrimps. Nautella had the ability to express virulence by stimulated by environmental factors, thereby increasing the susceptibility to infection, which may have a negative impact on healthy growth of shrimps. Pseudoalteromonas, Aliiroseovarius and Shimia were related to the pathogenicity of shrimp, which may be an important factor affecting the growth of shrimp. This research may provide valuable information on understanding the characteristics of intestinal bacteria in shrimp. These 
intestinal bacteria identified to provide important resources for further understanding the relationship between shrimp growth and intestinal bacterial. Together, these findings greatly enhance our understanding of the role of the intestinal bacteria in unsynchronized growth of shrimp.

\section{Acknowledgements}

This study was supported by Central Public-interest Scientific Institution Basal Research Fund, CAFS (2020TD30), Key Special Project for Introduced Talents Team of Southern Marine Science and Engineering Guangdong Laboratory (Guangzhou) (GML2019ZD0605); Hainan Provincial Natural Science Foundation of China (320QN359); China Agriculture Research System of MOF and MARA (CARS-48); Guangdong Basic and Applied Basic Research Foundation (2020A1515110200); Guangzhou Science and Technology Planning Project (202102020208); Central Public Interest Scientific Institution Basal Research Fund, South China Sea Fisheries Research Institute, CAFS (2020ZD01, 2021SD13).

\section{References}

Amato, K.R., Yeoman, C.J., Kent, A., Righini, N., Carbonero, F., Estrada, A., Gaskins, H.R., Stumpf, R.M., Yildirim, S., Torralba, M., Gillis, M., Wilson, B.A., Nelson, K.E., White, B.A., Leigh, S.R., 2013. Habitat degradation impacts black howler monkey (Alouatta pigra) gastrointestinal microbiomes. Isme Journal. 7, 1344-1353. https://doi.org/10.1038/ismej.2013.16

Amoah, K., Huang, Q., Dong, X., Tan, B., Zhang, S., Chi, S., Yang, Q., Liu, H., Yang, Y., 2020. Paenibacillus polymyxa improves the growth, immune and antioxidant activity, intestinal health, and disease resistance in Litopenaeus vannamei challenged with Vibrio parahaemolyticus. Aquaculture. 518. https://doi.org/10.1016/j.aquaculture.2019.734563

Angthong, P., Uengwetwanit, T., Arayamethakorn, S., Chaitongsakul, P., Karoonuthaisiri, N., Rungrassamee, W., 2020. Bacterial analysis in the early developmental stages of the black tiger shrimp (Penaeus monodon). Scientific Reports. 10. https://doi.org/10.1038/s41598-020-61559-1

Broekaert, K., Noseda, B., Heyndrickx, M., Vlaemynck, G., Devlieghere, F., 2013. Volatile compounds associated with Psychrobacter spp. and Pseudoalteromonas spp., the dominant microbiota of brown shrimp (Crangon crangon) during aerobic storage. International Journal of Food Microbiology. 166, 487-493.

Chaiyapechara, S., Rungrassamee, W., Suriyachay, I., Kuncharin, Y., Klanchui, A., Karoonuthaisiri, N., Jiravanichpaisal, P., 2012. Bacterial Community Associated with the Intestinal Tract of $P$. monodon in Commercial Farms. Microbial Ecology. 63, 938-953. https://doi.org/10.1007/s00248-011-9936-2

Duan, Y., Liu, Q., Wang, Y., Zhang, J., Xiong, D., 2018. Impairment of the intestine barrier function in Litopenaeus vannamei exposed to ammonia and nitrite stress. Fish \& Shellfish Immunology. 78, 279-288. https://doi.org/10.1016/j.fsi.2018.04.050

Duan, Y., Xiong, D., Wang, Y., Dong, H., Huang, J., Zhang, J., 2020. Effects of Microcystis aeruginosa and microcystin-LR on intestinal histology, immune response, and microbial community in Litopenaeus vannamei. Environmental Pollution. 265. https://doi.org/10.1016/j.envpol.2020.114774

Duan, Y., Wang, Y., Liu, Q., Dong, H., Li, H., Xiong, D., Zhang, J., 2019. Changes in the intestine microbial, digestion and immunity of Litopenaeus vannamei in response to dietary resistant starch. Scientific Reports. 9. https://doi.org/10.1038/s41598-019-429398

Gao, S., Pan, L., Huang, F., Song, M., Tian, C., Zhang, M., 2019. Metagenomic insights into the structure and function of intestinal microbiota of the farmed Pacific white shrimp (Litopenaeus vannamei). Aquaculture. 499, 109-118. https://doi.org/10.1016/j.aquaculture.2018.09.026

Hou, D., Huang, Z., Zeng, S., Liu, J., Wei, D., Deng, X., Weng, S., Yan, Q., He, J., 2018. Intestinal bacterial signatures of white feces syndrome in shrimp. Applied 
Microbiology and Biotechnology. 102, 3701-3709. https://doi.org/10.1007/s00253-0188855-2

Jiang, X., Peng, X., Deng, G., Sheng, H., Wang, Y., Zhou, H., Tam, N.F., 2013. Illumina Sequencing of 16S rRNA Tag Revealed Spatial Variations of Bacterial Communities in a Mangrove Wetland. Microbial Ecology. 66, 96-104. https://doi.org/10.1007/s00248013-0238-8

Lebel, L., Mungkung, R., Gheewala, S.H., Lebel, P., 2010. Innovation cycles, niches and sustainability in the shrimp aquaculture industry in Thailand. Environmental Science \& Policy. 13, 291-302. https://doi.org/10.1016/j.envsci.2010.03.005

Macias-Sancho, J., Poersch, L.H., Bauer, W., Romano, L.A., Wasielesky, W., Tesser, M.B., 2014. Fishmeal substitution with Arthrospira (Spirulina platensis) in a practical diet for Litopenaeus vannamei: Effects on growth and immunological parameters. Aquaculture. 426, 120-125. https://doi.org/10.1016/j.aquaculture.2014.01.028

Nahavandi, R., Hafezamini, P., Moeini, H., Jahromi, M.Z., Shamsudin, M.N., 2011. Population of bottleneck and microsatellite: An Analysis Based on genetic diversity of Wild Tiger Shrimp Penaeus monodon (Fabricius) in Malaysia. African Journal of Biotechnology. $10,16715-16719$.

Rungrassamee, W., Klanchui, A., Maibunkaew, S., Chaiyapechara, S., Jiravanichpaisal, P., Karoonuthaisiri, N., 2014. Characterization of Intestinal Bacteria in Wild and Domesticated Adult Black Tiger Shrimp (Penaeus monodon). Plos One. 9. https://doi.org/10.1371/journal.pone.0091853

Rungrassamee, W., Klanchui, A., Chaiyapechara, S., Maibunkaew, S., Tangphatsornruang, S., Jiravanichpaisal, P., Karoonuthaisiri, N., 2013. Bacterial Population in Intestines of the Black Tiger Shrimp (Penaeus monodon) under Different Growth Stages. Plos One. 8. https://doi.org/10.1371/journal.pone.0060802

Schloss, P.D., Gevers, D., Westcott, S.L., 2011. Reducing the Effects of PCR Amplification and Sequencing Artifacts on 16S rRNA-Based Studies. Plos One. 6. https://doi.org/10.1371/journal.pone.0027310

Sun, Y., He, M., Cao, Z., Xie, Z., Liu, C., Wang, S., Guo, W., Zhang, X., Zhou, Y., 2018. Effects of dietary administration of Lactococcus lactis HNL12 on growth, innate immune response, and disease resistance of humpback grouper (Cromileptes altivelis). Fish \& Shellfish Immunology. 82, 296-303. https://doi.org/10.1016/j.fsi.2018.08.039

Wang, C., Zhou, Y., Lv, D., Ge, Y., Li, H., You, Y., 2019. Change in the intestinal bacterial community structure associated with environmental microorganisms during the growth of Eriocheir sinensis. Microbiologyopen. 8. https://doi.org/10.1002/mbo3.727

Wu, S., Gao, T., Zheng, Y., Wang, W., Cheng, Y., Wang, G., 2010. Microbial diversity of intestinal contents and mucus in yellow catfish (Pelteobagrus fulvidraco). Aquaculture. $303,1-7$.

Xiong, J., Dai, W., Zhu, J., Liu, K., Dong, C., Qiu, Q., 2017. The Underlying Ecological Processes of Gut Microbiota Among Cohabitating Retarded, Overgrown and Normal Shrimp. Microbial Ecology. 73, 988-999. https://doi.org/10.1007/s00248-016-0910-x

Zeng, S., Huang, Z., Hou, D., Liu, J., Weng, S., He, J., 2017. Composition, diversity and function of intestinal microbiota in pacific white shrimp (Litopenaeus vannamei) at different culture stages. Peerj. 5. https://doi.org/10.7717/peerj.3986

Zhang, M., Sun, Y., Liu, Y., Qiao, F., Chen, L., Liu, W., Du, Z., Li, E., 2016. Response of gut microbiota to salinity change in two euryhaline aquatic animals with reverse salinity preference. Aquaculture. 454, 72-80.

Zheng, Y., Yu, M., Liu, J., Qiao, Y., Wang, L., Li, Z., Zhang, X.-H., Yu, M., 2017. Bacterial Community Associated with Healthy and Diseased Pacific White Shrimp (Litopenaeus vannamei) Larvae and Rearing Water across Different Growth Stages. Frontiers in Microbiology. 8. https://doi.org/10.3389/fmicb.2017.01362 